\title{
L'éthos « poilu » dans les lettres des soldats de la Grande Guerre : hétérogénéités énonciatives et stratégies discursives
}

\author{
Vicari, Stefano \\ Università degli studi di Genova \\ stefano_vicari@hotmail.it
}

\section{Introduction}

Des flots d'encre ont coulé pendant la Grande Guerre autour des "poilus », les qualifiant de courageux ou de virils, les imaginant heureux d'accomplir la tâche sublime de sauver leur patrie, joyeux et sans soucis. C'est ainsi que les articles de journaux, les éditoriaux, les romans, les cartes postales et les poèmes sublimaient l'image d'un poilu héroïque et alimentaient la création d'un imaginaire poilu, puisant fortement dans des idéaux et des valeurs patriotiques, valeurs qui plaisaient à la propagande et qui respectaient ainsi les contraintes imposées par la censure de l'époque (Forcade, 2005). Si le travail soigneux de la censure n'a permis presque aucun type de contestation explicite, il est néanmoins possible de repérer des traces d'un discours de contestation dans les lettres écrites par les poilus à leurs proches : notre hypothèse est en effet que le discours épistolaire des combattants contient, en dépit de la censure et de l'autocensure, les traces d'une contestation de la guerre et des conditions de vie inhumaines imposées aux soldats au fur et à mesure que le conflit se prolongeait dans le temps. Leurs lettres constitueraient ainsi le lieu d'une entreprise argumentative par laquelle les combattants entendaient non seulement entretenir des liens avec leurs proches mais aussi faire surgir leur point de vue sur l'expérience de la guerre à l'intérieur d'un «champ discursif» dominé par le discours de la propagande et de la mobilisation.

Dans cette étude, parmi les stratégies adoptées par les combattants, nous nous pencherons sur la construction discursive d'un éthos (Amossy, 2010 [2000]) "poilu» dans lequel les soldats se reconnaissaient. Pour ce faire, nous prendrons en compte certaines catégories d'observables qui nous paraissent constitutives du processus de construction de l'identité de l'éthos poilu et qui participent des mécanismes d'identification des voix contre lesquelles leurs critiques sont adressées : à savoir des stratégies de reconfiguration de l'éthos préalable auprès de la famille jusqu'à l'inscription en discours de commentaires portant sur leurs propres mots ou sur les mots des autres, en passant par des emplois du fort contesté « argot poilu » (Roynette, 2010). Nous montrerons ainsi les effets pragmatiques de la création de cette identité : quels sont les éléments qui, d'après les scripteurs, feraient partie de leur identité en tant que «poilus » ? Quelle image d'eux-mêmes construisent-ils en discours ? Et encore, cette image, répondelle à l'image relayée par les discours de la presse et des bulletins officiels ?

\section{Intérêt de l'analyse et remarques de méthode}

Le regain d'intérêt dont les correspondances des soldats font l'objet dans l'historiographie contemporaine (Cazals et Rousseau, 2001 ; Trévisan, 2003 ; Horne, 2005) et la publication récente d'un grand nombre de correspondances des poilus montrent clairement l'importance de ces témoignages afin de saisir l'expérience de la Grande Guerre dans ses aspects les plus intimes, dans le vécu de ceux qui l'ont combattue au front et donnent lieu à de nombreuses interprétations et études psychologiques différant 
sensiblement entre elles. Ces interprétations tournent autour de la question de connaître les motivations réelles de la participation guerrière de la part des poilus et que Horne reformule ainsi :

\begin{abstract}
Victimes d'une contrainte étatique et judiciaire, ou participants dans une violence consentie à l'égard de l'ennemi ? Motivés par une loyauté de groupe envers les camarades, ou mobilisés par un sentiment d'appartenance nationale? Les hypothèses abondent, sans forcément se contredire. (Horne, 2005 : 904).
\end{abstract}

Nous pensons que les outils de l'AD et, en particulier, les travaux d'Amossy (2010 [2000]) et de Maingueneau (1993) sur la construction de l'éthos en discours, nous permettent de mieux cerner cette question à partir des productions écrites des poilus eux-mêmes. La définition que Maingueneau donne d' « éthos discursif», à savoir :

Ce que l'orateur prétend être, il le donne à entendre et à voir : il ne dit pas qu'il est simple et honnête, il le montre à travers sa manière de s'exprimer. L'éthos est ainsi attaché à l'exercice de la parole, au rôle qui correspond à son discours, et non à l'individu «réel », appréhendé indépendamment de sa prestation oratoire. (Maingueneau, 1993 : 138)

nous permet en effet non seulement de situer la problématique de l'éthos au niveau des marques et de stratégies repérables à la surface du discours, mais aussi d'apporter des éclaircissements ultérieurs sur la relation entre genre de discours, champ discursif et marques discursives aptes à construire l'éthos dans les différents discours, comme le souhaite aussi Micheli (2011) :

\begin{abstract}
il ne suffit pas, en effet, d'affirmer que la construction discursive d'une image de soi est un phénomène qui traverse la quasi-totalité des genres discursifs, puis de nuancer une telle généralité en soulignant que l'élaboration d'un ethos est toujours en partie régie par des normes spécifiques à chaque genre. [...] quelles sont, au juste, les unités pertinentes pour saisir l'ethos dans la matérialité langagière, quels sont leurs différents modes de signifiance ? (Micheli, 2011 : en ligne)
\end{abstract}

L'hypothèse que nous allons essayer de démontrer à partir de l'analyse des lettres des soldats du front est alors que la prise en compte des renvois au champ discursif et au genre de discours auxquels les discours analysés appartiennent se révèle fondamentale pour le choix des observables pouvant témoigner de la construction en discours de l'éthos des locuteurs.

Notre analyse sera menée à partir d'un corpus constitué d'à peu près 1500 lettres écrites par des poilus pendant les années de guerre et contenues dans sept recueils de correspondances (voir la liste complète à la fin de la contribution). Bien que l'on compte que pendant les quatre années de guerre chaque soldat mobilisé ait écrit en moyenne une lettre par jour (Trévisan, 2003), notre corpus nous semble néanmoins représentatif de différentes typologies de scripteurs : du soldat de seconde classe à l'officier tous y sont représentés, même si dans des proportions variables (les deux volumes Paroles de Poilus et La dernière lettre sont transversaux et contiennent un nombre plus élevé de lettres rédigées par de simples soldats). Les différentes marques et stratégies seront présentées et analysées à partir des cas les plus fréquents et les plus représentatifs dans notre corpus.

\title{
3 Le dispositif énonciatif
}

Les dangers constants de mort et la vie souterraine dans la boue des tranchées ont favorisé non seulement la nécessité, de la part des soldats, d'entretenir des liens avec l'arrière, avec la vie civile, mais aussi la création d'une identité groupale, d'un nous par lequel tout poilu se fait le porte-parole de l'ensemble des combattants. C'est, du moins, ce que nous allons montrer par l'étude conjointe de certains emplois des pronoms personnels et de la mise en scène des altérités énonciatives. L'analyse nous permettra d'affiner ces affirmations tout en montrant les stratégies auxquelles les scripteurs ont recours pour exprimer leur malaise face à ce qu'on dit sur la guerre et sur les poilus. 


\subsection{Emploi des pronoms personnels}

\subsubsection{L'alternance je et nous}

Si le ton informel et l'appui elliptique sur des connaissances partagées entre destinateur et destinataire font en sorte que ces lettres appartiennent au genre de la correspondance intime (Adam, 1997), il est néanmoins remarquable de constater qu'au niveau de l'emploi des pronoms personnels le je du scripteur n'apparaît que très rarement dans les correspondances ou, du moins, les séquences textuelles présentent un va-et-vient incessant entre la première personne singulière et le nous :

[1] Départ 5 heures. A 7 heures passons la frontière - Enthousiasme (en Belgique) tabac, café, bière. Arrivons - Chimay (réception inoubliable en même temps tirailleurs algériens). Prenons la garde - Là tout nous est offert. (Olivier, p. 66)

[2] Chers parents,

Nous sommes toujours dans nos cantonnements de Ronchamps [...]. Nous sommes allés travailler aux premières lignes. [...] Nous avons paraît-il fait en 5 jours plus de travail qu'un corps d'armée en 4 mois. [...]. Nous allons probablement relever le 167e et le 168 e ces jours-ci. (Pensuet, p.73-74)

[3] C'est presque sans regret que j'ai quitté Paris, mais c'est la vérité. Que veux-tu, ¿'ai constaté, comme tous mes camarades du reste, que ces deux ans de guerre avaient amené petit à petit, chez la population civile, l'égoisme et l'indifférence et que nous autres combattants nous étions presque oubliés, aussi quoi de plus naturel que nousmêmes, nous prenions aussi l'habitude de l'éloignement et que nous retournions au front tranquillement comme si nous ne l'avions jamais quitté? (Biron, p. 104 ; nous soulignons dans tous les commentaires)

Ces extraits rendent compte de la construction d'une voix groupale qui permet aux scripteurs de se faire les porteurs d'un point de vue transcendant tout locuteur pour s'identifier avec celui de la communauté entière des poilus : ils se font en effet co-énonciateurs d'un discours, que tout poilu pourrait tenir, tantôt dans le but de présenter les différents déplacements des escouades (exemples [1] et [2]), plus fréquemment dans une visée critique et démarcative (l'exemple [3]). Dans le dernier commentaire, en effet, le nous entre dans une dialectique opposant les poilus, oubliés, aux civils, les Parisiens, considérés comme égoïstes et ingrats. L'emploi du nous configure donc un discours de groupe, celui-ci se constituant grâce à la prise de distance des autres, comme nous le montrerons également dans les paragraphes suivants.

\subsubsection{Les emplois de on}

Dans son analyse des valeurs du pronom « on », Rabatel (2001) soutient que ce pronom fonctionne tantôt comme pronom personnel tantôt comme pronom indéfini. Or, dans notre corpus et dans la presque totalité des exemples repérés, «on» est utilisé à la place du pronom embrayeur «nous». Les scripteurs entendent installer en discours un point de vue groupal, où les membres du groupe seraient capables de voir et d'écouter les mêmes réalités et d'éprouver les mêmes sentiments face à ces réalités tout en se cachant derrière une énonciation désubjectivisée par le « on ». De nombreux passages sont caractérisés par la présence massive du pronom « on » qui est souvent employé par les scripteurs dans des listes répertoriant les actions habituelles des tranchées ou de la vie de caserne :

[4] On nous a habillés ce matin [...]. Réveil à $6 \mathrm{~h}$ et demi : taratata. On boit le jus. $\underline{\text { On }}$ descend dans la cour, appel, etc. (Marquand, p. 22)

Nous allons partir à midi à $12 \mathrm{~m}$ sous terre, travailler à des abris souterrains, sans discontinuer. Il y a 6 hommes et un cabot par abri. C'est un travail de mineurs, car on boise de même. Puis à 5 heures on revient à nos cagnas pour la soupe. Nous en repartons à 8 heures jusqu'à 1 heure du matin, mais le soir on vide les sacs de terre sur le parapet, ce qu'on ne peut faire le jour. (Marquand, p. 120)

[5] On est, vous vous en doutez sans peine, complètement abruti. (Tanty, p. 180) 


\begin{abstract}
Le matin on fait quelque travail, sans autre utilité que de nous emm..., on est éreinté et, le temps aidant, on ne pense qu'à dormir [...]. On crève de soif, défense d'aller chercher de l'eau à coté, on écrit des lettres [...]. On ne dort que le jour. (Tanty, p. 166)

[6] Aprésant, on est au repos du 16. On a été pendan 15 jours dans les tranchées, c'étais durre. (Papillon, p.181)

On par[t] au moins 50 sur 100 que nous étions à l'hopital. On va directemen à Dijon [...]. On va passé la visitte aujourd'hui [...]. On sort à Dijon comme on veu. On est plus en prison. (Papillon, p. 231)
\end{abstract}

Les scripteurs insistent en effet à maintes reprises sur la monotonie de la vie de guerre, sur la réitération des actions, bref, sur le cafard qui les assaille : l'emploi de ce pronom donne parfaitement l'idée de la dépersonnalisation due aux conditions de guerre tout en devenant par là emblématique de la communauté poilue. Enchaînements paratactiques, surabondance de phrases courtes et suivant le même schéma $(\mathrm{On}+$ présent de l'indicatif + compléments), présence de détails minutieux dans les descriptions confèrent à certaines séquences un style décousu et réitératif, voire monotone, qui rend bien compte de la vie que les poilus conduisent et de leur expérience de la guerre : loin des beaux discours idéologiques de la propagande, ce caractère répétitif répond en effet au type de combat que les soldats sont en train de mener. Les innovations ayant profondément modifié les techniques du combat, les hommes ne s'affrontent plus en face à face : ils attendent pendant des heures, des jours, voire des semaines, avant d'organiser un assaut. La vie dans les tranchées, au lieu d'être caractérisée par la succession d'actions viriles et héroïques, devient le milieu où les soldats ont tout le temps d'explorer leur intimité et leur conscience. Ce nouveau type de combat favorise le repli du soldat sur lui-même, ainsi que la recherche intérieure d'une voie de fuite par l'écriture, bien que dans les journaux et sur les cartes postales l'image d'un poilu viril, qui lutte avec tout son corps, soit encore dominante et contribue à créer autour des combattants tout un imaginaire lié à la virilité, au lien avec le terroir et à la sauvegarde de la patrie.

Les emplois des pronoms montrent clairement la construction d'un éthos " poilu » communautaire, un nous/on collectif, pluriel englobant l'ensemble des combattants au front. Or, l'analyse des marques introduisant les hétérogénéités énonciatives nous permettra de délimiter ce groupe encore indéfini et de détailler les caractéristiques dans lesquelles il se reconnaîtrait.

\title{
3.2 Reconfiguration de l'éthos préalable et mise en scène des hétérogénéités énonciatives
}

S'il est vrai qu' « au niveau du fil du discours, localiser un point d'hétérogénéité, c'est circonscrire celle-ci, c'est-à-dire poser par différence, pour le reste de la chaîne, l'homogénéité ou l'unicité de la langue, du discours, du sens, etc. » alors les scripteurs effectuent «à travers chaque marque de distance, une opération de constitution d'identité pour le discours» (Authier-Revuz, 1984: 105). Dans notre corpus, une place importante est faite à la mise en discours de l'autre, sous toutes ses formes: du discours rapporté aux gloses métadiscursives, en passant par l'ironie, tous ces moyens semblent déployés par les scripteurs afin de restituer le prétendu vrai regard sur la guerre et, par là, rétablir une vérité des dires et des faits. La prise en compte de ces formes relevant d'un degré de densité métalinguistique variable nous permettra de repérer un espace de discours à travers lequel les soldats font surgir leur voix à l'intérieur du champ discursif représenté par l'ensemble des productions discursives circulant pendant le conflit mondial et, par là, de constituer un éthos à la fois de témoins de première ligne et de combattants.

\subsubsection{La dénonciation des discours « autres »}

Les poilus expriment souvent un certain malaise face aux mots employés par les « autres » car ils pensent savoir mieux décrire ce qu'ils connaissent mieux. Une véritable guerre des dires se déclenche alors dans les années de guerre où le champ adverse à celui des combattants est constamment reconfiguré dans les lettres ( $c f . \S 5)$. De la presse aux « bougres » des salons, les scripteurs ressentent et manifestent au fil des correspondances une nécessité incessante de se réapproprier les mots, ce qui apparaît comme une exigence presque vitale, bien que cette réappropriation, comme nous le verrons, soit difficile et le 
parcours pour la réaliser hardi. La presse et les discours de la propagande relayés principalement par les bulletins officiels sont les cibles préférées par les soldats : ces discours ne semblent pas représenter leur état d'âme, leur vraie condition de vie bien qu'ils prétendent en rendre compte :

[7] Ce sera très long et nous croyons tous être ici encore à Noël 1915. Prenez donc vos dispositions en conséquence. Ne croyez pas ce que peuvent vous faire espérer les journaux. C'est une guerre qui sera la ruine de tous les pays. (Bénard, p. 77)

[8] Papa, pour l'aviateur ce n'est pas où tu me dis, et tu ne le verras pas sur les journaux, attendu qu'on ne mentionne jamais nos pertes. (Marquand, p. 144)

Comme ces deux exemples le montrent, les questions auxquelles les soldats sont sensibles relèvent de la durée de la guerre et des récits des batailles : les lettres sont parsemées de commentaires de ce genre par lesquels les soldats invitent leurs proches à se méfier des discours officiels. La dévalorisation des discours, circulant dans la presse et dans les communiqués, passe également à travers la manière dont les dires « autres » sont inscrits en discours, comme on peut le constater dans les commentaires suivants :

[9] Vous vous faites des illusions monumentales, comme tous les civils ou lecteurs de journaux : Papa me désole de le voir s'attacher à cette idée que je puis faire quelque chose et qu'on soigne les malate. (Tanty, p. 172)

[10] Mon cher Maurice, tu dois lire dans les journaux nos hauts-faits de Carnoy. $\underline{\mathrm{Il} \text { y a }}$ beaucoup d'exagération et les combats ont été bien plus modestes qu'on ne le raconte. (Bénard, p. 118)

[11] On a parlé du 356e sur le journal Excelsior no. du 18 décembre, de ce que nous

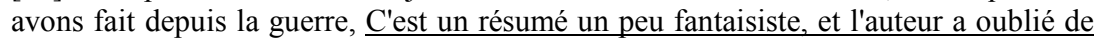
parler de notre randonnée de Nancy. (Papillon, p. 75)

Dans [9], le scripteur se limite à évoquer ce que les journaux écrivent par le biais d'une reprise interlocutive des mots du père: l'absence de citations directes est accompagnée d'un certain flou référentiel autour de la source dont les dires sont évoqués, à savoir « les journaux ». Cette source est donc reléguée à une posture de sousénonciation (Rabatel, 2004), tout comme cela arrive dans [10]. Dans le dernier commentaire ([11]), si la source est précisée, les dires ne sont pas vraiment rapportés, ils sont plutôt commentés par le scripteur qui en met en évidence le décalage par rapport aux faits. Ces quelques exemples sont représentatifs d'une tendance généralisée selon laquelle les discours ne sont ni vraiment rapportés ni attribués à une source particulière. Les scripteurs se limitent à les évoquer pour les juger négativement. Cela confirme à nos yeux que les dires, circulant dans la presse sur la guerre et les poilus, sont plutôt généralisés : difficile donc, pour eux, d'identifier une source, c'est bien là le champ discursif dans sa globalité qui est visé par ces commentaires. A cet égard, l'exemple suivant nous paraît emblématique de cette généralisation du discours de la propagande :

[12] Je termine, ne vous en faites pas, "on les aura" (les cheveux ras). (Papillon, p.

Repris entre guillemets, le slogan bien connu « 'on les aura'» fait l'objet d'une modalisation de la part du poilu : il s'agit d'un renvoi interdiscursif à des discours circulant dans le champ discursif autour de la Grande guerre, sans que le scripteur ait besoin d'en rappeler la source énonciative. Cependant, on ne peut pas vraiment établir ici le degré d'adhésion du scripteur à ces dires : s'agit-il d'une prise de distance ironique (ce que les guillemets et le jeu sur la rime « aura » - « ras » pourraient suggérer) ou bien d'un discours rapporté pour entretenir le moral et rassurer le destinataire ? Si ce dernier cas est ambigü, on peut affirmer que dans la plupart des exemples analysés montrent que la lettre constitue le moyen par lequel les poilus rétablissent leur vérité de témoins, dénoncent leur état d'âme et par là tentent de reconfigurer l'image que les civils se font d'eux d'après les « racontars » des journaux et du discours officiel à travers de nombreux renvois intradiscursifs et interdiscursifs. Les gloses analysées rendent bien compte de cette revendication d'un pouvoir dire en tant que seule prérogative des combattants, avec toutes les difficultés que ce dire comporterait, comme nous le verrons ci-dessous. 


\subsubsection{Le silence dit et montré : le poilu-témoin}

Dans bon nombre de lettres, les scripteurs insistent sur la difficulté que les civils ont à comprendre ce qui se passe véritablement sur le front : l'expérience guerrière se configure donc, dans les correspondances, comme un évènement qui dépasse les limites de la raison et de la compréhension humaines, au-delà du seuil du dicible :

[13] Hier soir seulement, pour faire la relève sur les pentes sud de Douaumont, au cours de la traversée du bois de la Caillette, ou plutôt de ce qui le fut, le 10e bataillon a beaucoup souffert; qu'il me suffise de vous dire que le lieutenant Verron a été tué, le capitaine Missaut blessé de nouveau, etc., etc.... [...] malgré tout mon courage, qui n'est pas amoindri, j'appréhende cette galopade à la mort. Il faut les vivre, ces minutes, pour en comprendre toute la tragique angoisse; tout sent le carnage : par ici, l'air est empesté d'une odeur de charnier. (Ninoret, p. 225-226)

Face au florilège de dires dans la presse, les poilus se trouvent à expliciter l'impossibilité de raconter la guerre et leurs conditions : ils disent le silence, le montrent de manière si constante dans l'ensemble du corpus qu'il est légitime de se demander quelles fonctions cette nécessité de parler du silence remplit. Dans la plupart des cas, les scripteurs thématisent cette impuissance par le biais de gloses métadiscursives du type de celles déjà étudiées par Authier-Revuz (1995) et Julia (2001). Il s'agit très fréquemment de gloses portant sur le décalage entre les mots et la réalité, comme dans les cas suivants :

[14] Nous avons tout de la bête féroce, $C^{\prime}$ est inénarrable, te dis-je! (Prouteau, p. 61)

[15] Pardonnez-moi de me plaindre, mais ce coup-ci j'y suis obligé car c'est une chose au-dessus de l'imagination, c'est à ne pas pouvoir dire. (Gilles, p. 93)

ou entre les mots et les sentiments éprouvés :

[16] L'affection ne se manifeste pas par les mots et ils sont si impuissants qu'on éprouve une espèce de pudeur à en parler ainsi ; je hais ces formules qui ne veulent rien dire, Il faut cependant que je vous le dise une fois : quoi qu'il arrive, la seule souffrance, pour moi, est la vôtre (Tanty, p. 66)

Les scripteurs se trouvent donc mal à l'aise avec les mots et les expressions toutes faites pour exprimer des états d'âme intimes, personnels : c'est justement « l'incessante reprise du déjà-dit » (Maldidier, 1990 : 89) qui permet alors aux scripteurs de s'en détacher pour exprimer avec encore plus de force et de vigueur leur souffrance : l'expression de la lacune lexicale ici, au lieu d'omettre une réalité, sert à rendre les sentiments éprouvés par les scripteurs plus tangibles. L'on est loin ici de l'image du poilu relayée par la propagande, riche en «formules qui ne veulent rien dire », d'un combattant disposé à tout pour sauver sa nation et ses territoires. Ces gloses montrent plutôt des hommes exténués, subissant un destin précaire qu'ils n'ont pas souhaité, et duquel ils voudraient s'enfuir. Les exemples suivants, où les scripteurs thématisent plus longuement l'impuissance des mots, confirment à nos yeux cette interprétation :

\section{[17] Les mots me manquent, je ne me retrouve plus, je suis tout hébété pour écrire ; la langue courante est impuissante. C'est un cauchemar atroce auquel je ne crois pas encore, un cauchemar qui va finir car il n'y a plus rien d'humain. (Tanty, p. 47) \\ [18] Tu vas croire que j'exagère, non. C'est encore en dessous de la vérité. On se demande comment il se peut que l'on laisse se produire de pareilles choses. Je ne devrais peut-etre pas décrire ces atrocités, mais il faut qu'on sache, on ignore la vérité trop brutale. (Pigeard, p. 54)}

Les poilus donnent ainsi l'idée d'avoir franchi une barrière entre le dicible et l'indicible, et soulignent à maintes reprises la distance ressentie comme incommensurable entre l'arrière et le front, dont seulement les poilus seraient et peuvent être les témoins. Le théâtre guerrier reste alors la prérogative des combattants, qui peuvent témoigner, de par leur présence dans les tranchées, des atrocités de cette guerre et de leur condition de victimes. Si le silence apparaît comme la conséquence ultime de l'état dans lequel ils se trouvent, il est également remarquable de constater que les soldats ressentent à plusieurs reprises le besoin de revenir sur leur propres mots afin de spécifier le sens avec lequel ils les emploient. L'on retrouve alors des gloses portant sur la non-coïncidence des mots à eux-mêmes, à travers lesquelles les 
scripteurs insistent sur la nécessité de prendre à la lettre ce qu'ils affirment et par là restituer aux mots toute leur épaisseur sémantique :

\author{
[19] La position de batteries allemandes se situe avant l'étang de Forges, où \\ commence, derrière, l'enfer de 304. Je ne dis pas enfer à tort car c'est ici que \\ commence le royaume des tirs de barrage. (Bordeching, p. 109) \\ [20] On est littéralement enduit de boue. (Papillon, p. 120)
}

Il n'est pas rare de rencontrer des cas de reformulations par lesquelles les poilus redéfinissent des mots susceptibles maintenant d'assumer d'autres sens (de [21] à [23]) et/ou de référer à de nouvelles réalités (de [24] à [26]) :

[21] Nous nous reposons aujourd'hui. Ce qu'on appelle se reposer, c'est rester un peu en arrière prêts à partir s'il le faut. (Olivier, p.118)

[22] On dit en repos, mais réellement le temps se passe à faire des corvées de bois pour les cuisines, pour faire des abris, puis le nettoyage et nous faisons aussi une petite marche militaire pour nous dégourdir et ne pas être trop mou quand il faudra repartir. (Olivier, p. 230)

[23] Chef de poste au patelin, où nous sommes au soi-disant repos. (Marquand, p. 145)

[24] Ce n'est pas une guerre qui se passe actuellement, c'est une extermination d'hommes. (Papillon, p. 81)

[25] Ce n'est plus une guerre, c'est une boucherie. (Marquand, p. 62)

[26] Jamais, depuis que je suis au régiment, je ne me suis senti de cette humeur comment l'appeler au juste? (Tanty, p. 58)

$\mathrm{Au}$ niveau des marques formelles, ces gloses impliquent des degrés de subjectivité variables: si les marques affichant le je du scripteur ne manquent pas («Je ne dis pas enfer à tort »), les gloses présentant des structures désubjectivisées sont plus nombreuses (emplois du « on », du présentatif « c'est » et des infinitivisations) : il s'agit bien là de la construction d'un discours qui vaut pour tous les poilus partageant la même expérience. Dès leur entrée en guerre, les soldats se trouvent en effet confrontés à de nouvelles réalités et à de nouveaux états d'âme : les «vieux» mots ne semblent plus suffire à décrire cette expérience, d'où la nécessité de réduire la distance, surtout linguistique, pour pouvoir témoigner. Ces gloses montrent donc l'importance de la langue utilisée dans le témoignage : les mêmes mots semblent s'enrichir de nouveaux sens, recouvrir de nouvelles réalités et cela oblige les scripteurs à une activité métalinguistique incessante.

La prise en compte de ces marques révèle un paradoxe entre la nécessité de parler et l'impossibilité de choisir les bons mots pour raconter avec précision, pour communier avec les destinataires. Au fil de cette recherche récurrente des mots «justes », l'on peut entrevoir le rapport intime que les poilus entretiennent avec la guerre, avec les mots de la propagande, etc. ainsi que la double exigence de témoigner leur propre guerre et de crier leur isolement, et cela afin de se réapproprier un espace intime ; une identité qui passerait par la réappropriation des bons mots, mais ressentie comme perdue.

\title{
4 Mots d'argot ou le poilu-combattant qui ne s'en fait pas
}

Les lettres analysées, à l'encontre de nos hypothèses initiales, ne présentent pas un grand nombre de mots qu'on pourrait reconduire à ce que l'on appelle traditionnellement « argot poilu ». Cette langue, dont la fonction est d'abord cryptique, puis identitaire (Calvet, 2007), semble plutôt être reléguée à la communication entre pairs. En 1916, en effet, les poilus montrent un certain malaise face à la grande diffusion de l'argot dans la presse : c'est ce que Roynette confirme lorsqu'elle écrit que le discours combattant est « sensible dès 1916 et soucieux de reprendre le contrôle d'un vocabulaire dont les soldatset c'est là toute l'ambigüité - ont favorisé en 1914-1915 le glissement, déjà amorcé avant-guerre, vers les civils » (Roynette, 2010 : 30). Ressenti comme l'un des facteurs majeurs de distinction sociale, l'argot n'appartient pas aux civils, comme les poilus ne cessent de le souligner : 
[27] J'ai fini le bouquin de Barbusse qui est très bien ; c'est très réaliste et très bien rendu. A mon avis c'est le seul bouquin qu'on ait fait de potable sur les Poilus (à part les réflexions de la fin qui sont fausses dans la bouche des hommes). (Marquand, $\mathrm{p}$. 214)

Tout en admettant la vraisemblance du roman, le scripteur tient à rappeler la distance, qui passerait par les mots « dans la bouche des hommes », entre eux et l'arrière. Or, cela dit, il nous semble que les quelques cas d'insertion de mots d'argot font partie des moyens langagiers adoptés par les poilus afin d'insister sur cette distance et, en contrepartie, créer une identité poilue, tant il est vrai que « Si on nomme pour l'autre, en nommant, on donne également une image de soi : on se montre » (Moirand, 2007 : 36 ; ital. et gras de l'auteure). Les cas repérés relèvent en effet de dénominations par lesquelles les poilus entendent appréhender et rendre compte de leurs activités et/ou de leurs états d'âme :

[28] On ne mange pas bien, on ne touche pas de vin, bref, c'est la mouise (Marquand, p. 94)

[29] Nous, on a du temps à autre la visite des taubes [avions allemands] et c'est tout. (Marquand, p. 138)

Les mots d'argot peuvent donc être introduits sans qu'aucune marque ne soit explicitée par les scripteurs, comme le montrent les exemples ci-dessus, mais, plus fréquemment, ils font l'objet d'une modalisation autonymique : de la simple marque typographique (guillemets) à de véritables énoncés définitoires et reformulations :

[30] Ce soir "on met encore les cannes" pour aller encore plus près des tranchées (Marquand, p. 118)

[31] Pour ton invitation à manger de la morue, je t'en remercie sincèrement, j'ai mieux que ça : nous nous mangeons de la "jambe de Boches". La jambe de Boches est naturellement de la viande cuite et saucissonnée comme une cuisse humaine. (Olivier, p. 224)

Dans [31] le syntagme nominal argotique est non seulement mis entre guillemets, mais aussi il est complété par une définition spontanée dont le modèle est $X$ est remplissant dans ce contexte une fonction didactique : afin d'assurer l'intercompréhension avec la femme aimée, Olivier fournit une définition du terme emprunté à l'argot des tranchées. Dans d'autres cas, plus nombreux, c'est la glose métadiscursive qui, au lieu de rendre le discours plus transparent, propose des reformulations en argot :

[32] Ils [les Allemands] ne se réveillent que la nuit pour nous envoyer des obus. Ces machins-la ça siffle, mais c'est rare que ça fasse du mal. C'est ce qu'on appelle des pets de lapin. (Olivier, p. 126-127)

Le reformulant est ici plus opaque que le reformulé et l'intention pédagogico-didactique est moindre, sinon absente : cette glose répond plutôt à la volonté de dédramatiser le récit de la guerre, ce à quoi la trivialité de l'expression concourt pleinement. L'intention ludique se manifeste également dans une certaine créativité lexicale, comme le commentaire suivant le montre bien :

[33] Bref, nous revoilà dans la cambouise. (Marquand, p. 139)

La création du mot-valise à partir de la contraction de deux mots d'argot facilement reconnaissables (cambouis et mouise) révèle ainsi comment l'argot remplit une fonction de divertissement. Si ces exemples montrent que de nombreux mots d'argot relèvent de la description des conditions de vie dans les tranchées et des actions militaires, l'autre champ sémantique recouvert par l'argot dans les lettres concerne la sphère des sentiments et des états d'âme, surtout dans le but de rassurer les proches :

[34] Je ne me fais pas de bile; quand on remontera au feu, ne vous en faites pas non plus. (Marquand, p. 72)

[35] Ce sont eux qui font tout le travail et moi je n'en fous pas la rame. (Marquand, p. 208)

[36] Ne vous faites pas de bile, je ne m'en fais guère. (Papillon, p. 29)

[37] Je suis toujours dans les tranchées. On finit par s'y faire. (Papillon, p. 62) 
Ces exemples nous montrent l'image d'un groupe (identifié par les « on ») qui, au fur et à mesure que le conflit avance, s'habitue aux conditions de vie et aux tâches imposées par les officiers : les scripteurs manifestent la volonté de rassurer leurs proches en créant le prototype du poilu combattant qui ne s'en fait pas et qui accepte bon gré mal gré sa nouvelle vie au front. Ces exemples contribuent donc à la «construction d'une image de soi dans laquelle les mots et la manière dont les soldats s'expriment deviennent une allégorie de l'âme de la nation » (Roynette, 2010 : 19) au détriment du langage des autres, qu'ils soient allemands ou américains :

[38] Les Boches doivent en avoir assez du bombardement qui dure depuis 12 jours [...] nous on se charge bien de leur montrer ce que valent les «Franzouzes». (Pensuet, p. 221)

[39] Cette nuit, on a fait un prisonnier, c'est un Saxon, il était pris dans les fils de fer, il gueulait "Kamarad Franzious". (Papillon, p. 82)

[40] Il fallait entendre les jurons d'outre-Manche [sic, outre-Atlantique] dans la nuit, sous les branchages : « Go home !, Pull up !, All right ! . Mais tout cela dit d'un ton flegmatique à l'excès. [...]. Ces 2 derniers [deux américains] parlent un anglais très doux, sans accentuation gutturale; on y retrouve un peu la douceur et le « fili » des langues latines. [...] Nous remarquons ici la différence sensible de courtoisie des Américains et des Anglais. Les premiers sont beaucoup plus froids et moins accueillants. A un service rendu, ils répondraient de même, mais ne diront jamais : «Merci », D'ailleurs, entre eux, ils s'abordent sans formule de politesse aucune. (Marquand, p. 277)

Des simples marques typographiques (exemples [38] et [39]) à de véritables commentaires métalinguistiques (exemple [40]), ces commentaires montrent bien le rôle assumé par la langue dans le conflit, en tant que marque de distinction identitaire : les mots et les manières de parler des autres, repris et disqualifiés, correspondent à la même volonté de poser des frontières entre eux, les poilus, et les autres, qu'ils soient les ennemis ou les alliés.

\section{L'identité par les antithèses : les poilus et les Boches, les civils et les officiers}

Comme Branca-Rosoff (1990) l'a déjà remarqué, le discours épistolaire des poilus révèle l'emploi massif $\mathrm{d}$ 'antithèses portant principalement sur l'opposition faite entre eux-mêmes et leurs ennemis, les Boches. Cela dit, au fil des correspondances, les antithèses repérées portent également sur d'autres composants de la société française, internes et externes à l'armée, tels que les officiers et les civils de l'arrière.

\subsection{Les poilus et les Boches}

L'antithèse opposant les poilus aux Allemands ne se limite pas au choix de la dénomination «Boche » qui s'installe de manière définitive à partir des premiers mois de la guerre et qui perd au fur et à mesure la connotation négative qui lui était associée au début: d'autres désignations, certes plus éphémères, contribuent à installer en discours une dévalorisation de l'ennemi :

[41] As-tu lu le récit de l'attaque de Wambrechies et son bombrdement. Pauvre église, elle était si élégante, il fallait que ces animaux-là se vengent de se voir battus là comme partout. (Olivier, p. 148)

[42] Il y avait deux cochons dans le tas; ils n'ont pas été épargnés; pour ma part j'en ai abattu 2 comme au tir au lapin. (Pensuet, p. 226)

[43] Nous attendons la réponse des Boches avec sérénité ; elle ne tardera guère car ces sales oiseaux n'ont guère l'habitude de se laisser faire. ( Marquand, p. 136)

[44] Ces cochons-là croient toujours dans la victoire. (Papillon, p. 118)

Ces désignations dénigrantes se présentent comme des reprises anaphoriques (elles sont en effet presque toujours introduites par des démonstratifs) du terme "Boche », celui-ci étant sans doute ressenti comme trop neutre par rapport au jugement de type évaluatif exprimé par les désignations proposées. En effet, 
bien qu'il s'agisse d'anaphores, et donc présentées par les scripteurs comme des synonymes de « Boche », ces désignations rendent compte du processus de brutalisation des ennemis, considérés comme de véritables bêtes : sales oiseaux, cochons, animaux ne font qu'alimenter un imaginaire des Allemands lié à la dégradation, à la férocité ainsi qu'à la lâcheté et à l'infériorité militaire :

[45] Nos aéros font toujours la chasse aux Boches qui fuient comme des "péteux". (Marquand, p. 131)

[46] Les Boches faiblissent, leur artillerie est loin d'égaler la nôtre. Il faut espérer que ce carnage finira bientôt. (Papillon, p. 115)

Inutile de rappeler ici les liens interdiscursifs que ce véritable paradigme désignationnel (Mortureux, 1993), portant sur l'animalisation des Allemands, entretient avec les discours de la propagande et de la presse officielle: il suffit d'observer certaines images relayées sur les cartes postales pour se rendre compte de l'ampleur du phénomène, surtout à partir de la deuxième année de guerre (Mosse, 1999).

\subsection{Les poilus et les civils}

Si les Boches occupent une place privilégiée dans l'imaginaire de toute la société française, il est remarquable de constater la présence d'une antithèse, qui naît au fur et à mesure avec l'avancement de la guerre, entre les civils et les combattants au front : les expériences étant si exclusives qu'elles ont permis la diffusion d'une culture commune seulement aux poilus (Rheault, 2004) ainsi qu'un ressentiment qui se décline à travers des marques discursives différentes, comme les commentaires suivants le montrent :

[47] Depuis juillet nous nous sommes séparés ; et tandis que, grâce à l'héroïsme de nos troupes, vous pouvez continuer vos études dans la quiétude d'une ville préservée de l'invasion, je vis, pour ma part, au milieu d'horreurs inimaginables. (Boulle, p. 85) [48] Je croyais que vous vous rendiez mieux compte de la réalité et que je pouvais vous raconter ainsi toute la vérité. Et, je m'en rends compte peu à peu, je n'ai déjà plus la mentalité de la vie civile.. Songez, et vous avez dû le voir dans mes dernières lettres, que le repos et la sécurité dont nous parlions sont très relatifs. A vivre perpétuellement cette vie, on s'accoutume à l'envisager et à penser à ses incertitudes et à en parler. (Tanty, p. 116)

Les tons sont ici certes plus mitigés que dans les exemples pourtant sur les Boches: dans ces commentaires c'est le sentiment d'une distance qui prédomine, d'une incommunicabilité entre deux mondes qui ne vivent plus la même réalité, les nouveaux événements et les conditions de vie étant inconcevables pour ceux qui ne les vivent pas sur leur peau :

[49] Celui qui n'y a pas été ne peut pas se faire une idée de ce que c'est qu'être si longtemps debout et autant dire sans dormir, sous la pluie, le vent, le froid. (Olivier, p. 268)

[50] Il faut y avoir passé pour savoir ce que c'est. Assurément quand vous autres, vous lisez le journal, tranquillement au coin du feu vous vous dites : ah ces pauvres soldats. (Olivier, p. 305)

[51] Les braves civils s'apprêtent à partir à la campagne, les militaires s'apprêtent à partir en campagne: simple question de préposition après tout. Et cependant, de mettre l'une à la place de l'autre, quelle différence !Allez donc blaguer la grammaire après cela, quand un si petit mot produit tant de changements! (Tanty, p. 42)

Dans ce sous-corpus, les scripteurs insistent sur les limites d'un quelconque témoignage, sur l'impuissance des mots à décrire les dures conditions imposées par le conflit et sur la nécessité de constater avec ses propres yeux pour pouvoir parler, témoigner. Encore une fois, l'on retrouve donc la construction discursive d'une communauté de combattants qui se fait témoin d'évènements inimaginables : les désignations des poilus et des civils renvoient en effet à des noms collectifs, (nos troupes, les militaires VS vous, vous autres, les braves civils) ayant l'effet non seulement de styliser en quelques traits des communautés, certes non homogènes, mais aussi de créer une antithèse binaire qui nous semble parcourir l'ensemble des correspondances et qui voit s'opposer d'un côté les combattants, de l'autre les Allemands, les civils et, enfin, les officiers. 


\subsection{Les poilus et les officiers}

Les chefs font l'objet de nombreuses critiques, déjà à partir de la deuxième année du conflit, de la part des scripteurs qui dénoncent souvent l'incompétence des officiers et leur lâcheté sur le champ de bataille :

[52] $\mathrm{Tu}$ verras que ce sont eux [les chefs] qui ont remporté les victoires, comme l'autre en se mettant derrière les meules et en criant " en avant, à la baïonnette". Pour cela il ne faut qu'une bonne gueule, plus on hurle, mieux ça vaut. Enfin... Nous savons que nous avons fait tout notre devoir et beaucoup mieux qu'eux. Cela nous suffit. Que nous importe les médailles et les Légions d'honneur. Nos médailles à nous, soldats, ce seront nos blessures et si nous ne sommes pas blessés, nous aurons dans les yeux la flamme des Lebel. Assez. (Olivier, p. 251)

L'antithèse dans cet exemple, comme dans d'autres, se construit à partir de l'emploi des pronoms personnels eux, les chefs et nous, les poilus : à la lâcheté des uns correspondrait la valeur des autres. Bons parleurs, les officiers ne supporteraient les épreuves des tranchées que dans les discours qu'ils proféreraient :

[53] J'ai eu l'occasion d'entendre un conférencier, un écrivain très connu : Marc Sangnier, directeur du Sillon. Beau parleur, ciseleur de "fleurs de rhétorique", il nous débita pendant 2 heures la thèse gouvernementale sur l'origine des causes de la guerre. C'est le bourrage de crane officiel et je ne puis subir ce "gavage" que pendant 30 minutes [...]. Je passe sur cette manifestation forcément bien vue de tous nos officiers, lecteurs de l'Echo de Paris. (Marquand, p. 270)

Aux beaux discours du bourrage de crâne les poilus répondent avec des actions valeureuses directement dans les tranchées. L'antithèse se développe également au niveau des dénominations attribuées aux officiers, même au-delà de celles utilisées dans l'argot (Dauzat, 1918) :

[54] Si jamais Papa était mobilisé et que j'en rencontre un, même service auxiliaire, de ces jeunes paltoquets [les généraux], je serais bien capable de lui casser proprement la gueule; et je ferais comme je le dis, malgré mon caractère pacifique. (Marquand, $\mathrm{p}$. 188)

[55] On se "marre" intérieurement lorsque l'un de ces beaux messieurs entre en conversation avec nous! Ils ne risquent guère de nous contredire, car ils répètent les mots techniques et ajoutent : "Oui, oui, je sais!", de l'air entendu du monsieur au courant. Mais...il y a loin, bien loin de là. (Pensuet, p. 290)

[56] Ces Messieurs jugent que nous vivons comme eux, touchant double et triple ration et faisant venir de Reims, par cyclistes et voitures, tout ce qu'il faut. (Tanty, p. 99)

Ces dénominations sont volontiers précédées de démonstratifs qui ne remplissent aucune fonction anaphorique ou déictique : ils renvoient plutôt à des référents génériques dont la fonction, selon GaryPrieur, est celle d'établir «entre le locuteur-auteur et le lecteur une forme de connivence, puisqu'elles impliquent qu'ils partagent l'expérience d'une classe empirique » (Gary-Prieur, 1998 : 49).

Dans certaines correspondances, à l'officier responsable est même attribué un sobriquet, comme c'est le cas pour Dérange-tout, dont Tanty n'hésite pas à en mettre en évidence la trivialité des comportements et des manières de parler:

[57] Et nous resterions là, plantés comme des larbins, l'air ahuri, si le colonel, apercevant un aéro, ne nous disait de rentrer aux gourbis ce que - excusez l'expression - Dérange-tout traduit en son langage : " Allez ouest, là ! Secouez le panier à crottes. » Il affectionne tant ce langage. (Tanty, p. 144)

Le dernier procédé qui nous semble constitutif de l'antithèse avec les officiers relève du discours rapportant directement les jugements des camarades combattants sur leurs chefs, comme dans les deux cas suivants :

[58] Papa m'écrit la visite de Painlevé au front. Ecoutez l'impression des simples poilus : "C'est du chiqué! Il veut jouer à son petit Kerensky". Ont-ils tort? Pour moi je 


\begin{abstract}
vous assurerai qu'il me semble invraisemblable que M. le Ministre en personne ait assisté à une attaque dans les rangs des poilus, attendu que...nos officiers n'y assistent même pas...et pour cause! Ils nous font pitié avec leurs essais de machine remontemoral!! ( Marquand, p.204)

[59] J'avais trouvé quelques beaux articles dans la dernière Revu, notamment une critique de la conduite de Girard Maurice, Celui-ci avait écrit : "Mon devoir de citoyen passe avant mon devoir militaire", J'ai soumis la phrase en question au Soviet le soir à souper. Elle a déchainé : "la pôvre", les arguments vitupérents de quelque sectaire socialiste, dont la fougue fut bientôt calmée sous la douche de quelques froides paroles d'un "terrible torial" [territorial]. (Marquand, p. 262)
\end{abstract}

La source des dires rapportés, cette fois en citation directe et en guise de support des dires du scripteur, est représentée par une voix groupale, celle des poilus, et montre encore une fois l'expression d'un point de vue unanime et partagé sur les chefs.

\title{
6 Conclusion
}

S'il est désormais un topos de la littérature du domaine (Amossy, 2010 [2000]) celui d'affirmer l'impossibilité de délimiter les marques pouvant rendre compte de la construction de l'éthos, la richesse et la variété des moyens langagiers et discursifs adoptés par les combattants dans leurs correspondances afin de créer un éthos «poilu », nous permettent de formuler, en guise de conclusion, quelques éléments de réponse à notre hypothèse concernant les modalités dans lesquelles l'éthos s'inscrit dans la matérialité langagière et discursive. Il nous semble en effet que nos analyses suggèrent que tout éthos apparaît strictement lié aux contraintes non seulement du genre discursif, mais aussi à celles imposées par le champ discursif auquel les textes étudiés appartiennent. Nous avons donc pu constater à maintes reprises comment (1) les contraintes d'ordre interdiscursif (les discours autres, qu'ils soient officiels ou de la presse), (2) le poids des nouvelles réalités auxquelles les scripteurs étaient confrontés et, enfin, (3) les contraintes propres au genre épistolaire (l'attention constante aux interlocuteurs s'exprimant dans la volonté de les rassurer) contribuent à créer la nécessité, de la part des scripteurs, de montrer leur identité, de poser des frontières entre eux et les autres et, par là-même, d'inscrire un discours de contestation mal supporté par la censure. Dans ces écrits, les nombreux renvois intradiscursifs et interdiscursifs repérés, les exemples d'hétérogénéité énonciative où les mots s'entremêlent à des silences, à des reformulations et à des lacunes lexicales, les emplois de dénominations et désignations issues de l'argot et, enfin, les antithèses corroborant la constitution d'une voix groupale ne seraient donc constitutives de la construction de l'éthos des scripteurs que dans les conditions d'écriture propres à ces lettres : insérées dans un vaste réseau de renvois continus aux discours officiels et de la presse, tout comme à ceux des proches et de la société civile, les correspondances racontent en effet toute la tragédie personnelle et humaine ressentie par les soldats, leur ressentissement envers la guerre et leur bésoin impérieux de dire le vrai. Nous nous proposons de poursuivre ces quelques réflexions et d'affiner nos hypothèses à la lumière de comparaisons avec d'autres corpus afin de creuser davantage les critères de choix des observables linguistiques aptes à rendre compte de la construction discursive de l'éthos dans les différents genres textuels.

\section{Corpus}

Bénard, H.(1999). De la mort, de la boue, du sang. Lettres de guerre d'un fantassin de 14-18, Grancher : Paris. Foch, F. (éd.) (1922). La dernière lettre écrite par des soldats français tombés au champ d'honneur 1914-1918. Flammarion : Paris.

Guéno, J.-P. (éd.) (1998). Paroles de Poilus Lettres et carnets du front de 1914 - 1918. Librio : Paris.

Marquand, A. (2011). Et le temps, à nous, est compté" : Lettres de guerre (1914-1919). C'est-à-dire : Forcalquier.

Olivier, G. (2008). Afin de ne jamais oublier, Vie et mort d'un poilu hérö̈quement ordinaire, Gaston Olivier, soldat au 274e RI. Editions Anovi : Le Chaufour.

Papillon, L. (2003). "Si je reviens comme je l'espère ». Lettres du Front et de l'Arrière. 1914-1918. Grasset : Paris. Pensuet, M. (2010). Écrit du front, Lettres de Maurice Pensuet, 1915-1917. Tallandier : Paris.

Tanty, E. (2002). Les violettes des tranchées, Lettres d'un Poilu qui n'aimait pas la guerre. Editions Italiques: Paris. 


\section{Références bibliographiques}

Adam, J.-M. (1997). Les genres de discours épistolaire. De la rhétorique à l'analyse pragmatique des pratiques discursives. Pratiques, 94, 37-53.

Amossy, R. (2010 [2000]). L'argumentation dans le discours. Armand Colin : Paris.

Authier-Revuz, J. (1984). Hétérogénéité(s) énonciative(s). Langages, 73, 98-111.

Authier-Revuz, J. (1995). Ces mots qui ne vont pas de soi. Boucles réflexives et non-coïncidences du dire. Larousse : Paris.

Branca-Rosoff, S. (1990). Conventions d'écriture dans la correspondance des soldats. Mots. Les langages du politique, 24, 21-35.

Calvet, J.-L. (2007). L'argot. PUF : Paris.

Cazals, R., Rousseau, F. (2001). 14-18, le cri d'une génération. Privat : Toulouse.

Dauzat, A. (1918). L'argot de la guerre, d'après une enquête auprès des officiers et soldats. Armand Colin : Paris.

Forcade, O. (2005). Voir et dire la guerre à l'heure de la censure. Le Temps des médias, 4, 50-62.

Gary-Prieur, M.-N. (1998). La dimension cataphorique du démonstratif. Etude de constructions à relative. Langue française, 120, 44-51.

Horne, J. (2005). Entre expérience et mémoire : les soldats français de la grande guerre. Annales. Histoire, Sciences sociales, $5,903-919$

Julia, C. (2001). Fixer le sens. La sémantique spontanée des gloses de spécification du sens. Presses de la Sorbonne Nouvelle : Paris.

Maingueneau, D. (1993). Le Contexte de l'œuvre littéraire. Enonciation, écrivain, société. Dunod : Paris.

Maldidier, D. (éd.) (1990). L'inquiétude du discours. Textes de Michel Pecheux choisis et présentés. Editions des Cendres : Paris.

Micheli, R. (2011). Amossy, Ruth. 2010. La présentation de soi. Ethos et identité verbale (Paris : PUF). Argumentation et Analyse du Discours, 7, en ligne : http://aad.revues.org/1226 consulté le 09/03/12.

Moirand, S. (2007). De la nomination au dialogisme : quelques questionnements autour de l'objet de discours et de la mémoire des mots. In Cassanas A., Demange A., Laurent B. et Leclerc A. (sous la dir.). Dialogisme et nomination. Publications de l'Université Montpellier : Montpellier, 27-61.

Mortureux, M.-F. (1993). Paradigmes désignationnels. Semen, 8, 124-141.

Mosse, G. L. (1999). De la Grande Guerre au totalitarisme. La brutalisation des sociétés européennes. Hachette Littératures : Paris.

Rabatel, A. (2001). La valeur de "on" pronom indéfini/pronom personnel dans les perceptions représentées. L'information grammaticale, $88,28-32$.

Rabatel, A. (2004). L'effacement énonciatif dans les discours rapportés et ses effets pragmatiques. Langages, 156, 317.

Rheault, S. (2004). Rhétorique de la rupture dans les textes de poilus. Rhetor, I, 1-14.

Roynette, O. (2010). Les mots des tranchées. L'invention d'une langue de guerre 1914-1918. Armand Colin : Paris.

Trévisan, C. (2003). Lettres de guerre. Revue d'histoire littéraire de la France, 103, 331-341. 